\section{BOOK REVIEWS}

\section{The pick of the crop}

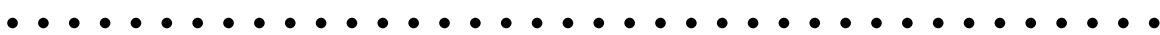

Annual Review of Genomics and Human Genetics, volume 4, 2003

Edited by Lander E, Page D, Lifton R. Annual Reviews, Palo Alto, CA, USA

..........

Andrew John Walley

European Journal of Human Genetics (2005) 13, 990.

doi:10.1038/sj.ejhg.5201424

$\mathrm{T}$ I he Annual Reviews series aim to provide timely, concise and authoritative reviews covering relevant topics within a single field. With the rapid growth of genetics and genomics in the last decade, Annual Reviews began a series of volumes covering this field and this review covers volume 4 . I would not attempt to review in-depth each and every chapter, but will try to convey my overall impressions of the utility of this book from the perspective of someone who has been working in the field of human genetics, on both monogenic and complex diseases, for over 10 years now.

The first thing that struck me on looking at the chapter index was the breadth of articles, covering a wide range of different topics. Subjects include the theory and practice of gene annotation, animal models of disease, model organism genomes, forensics, human genetic diversity and even clinical therapy for genetic disease. However, the majority of the articles concern the genetics of human disease, and are written by significant figures in those fields of study. As such, this volume continues the tradition of Annual Reviews in presenting high-quality articles summarising the current state of the art in specific areas of interest. For example, within my own area of interest in genetic analysis of complex traits, the article on diabetes mellitus by Florez, Hirschhorn and Altshuler is a clear summary of research in that field and how it has helped drive the analysis of complex traits in general. Of necessity, this review volume suffers from the perennial problem that there may not be a review of your particular field, but the range of subjects usually leads to some interesting articles for anyone working in genetics and genomics.

However, an important problem has become apparent with this volume. Annual Reviews also publishes a Genetics volume and there seems to be a grey area where the two series are now overlapping. Initially, ARGHG stayed within the boundaries of human genetics and genomics, but it has recently moved to cover non-human genomics and animal models. To the human geneticist, these chapters are probably only of passing interest and seem out of place, being more suited to the Annual Review of Genetics. Other subjects do not really seem to have a place in this series at all. A chapter debunking 'Creationism and Intelligent Design' is to be applauded in principle, but it seems inappropriate in a book aimed at reviewing current scientific progress. Equally, the review of 'Enzyme Therapy for Lysosomal Storage Disease' is wellwritten but seems only peripherally relevant, being predominantly about clinical treatment practice. Clearly, identification of the clinical relevance of the science is important in a basic science review, but this is not the place for a clinical review article.

In summary, this continues the high standards of the Annual Reviews and is to be recommended in principle to anyone with an interest in Genomics and Human Genetics as a good place to look for insightful articles covering a range of different fields of research. However, it is becoming increasingly clear that monthly review journals, such as Nature Reviews Genetics, are providing stiff competition, being more up to date and offering side benefits such as news and opinion articles, and it is going to be interesting to see how Annual Reviews respond to this challenge in the future

AJ Walley is at the Section of Genomic Medicine, Division of Medicine, Hammersmith Hospital, Du Cane Road, London W12 ONN, UK. E-mail: a.walley@imperial.ac.uk

\title{
A most welcome new edition in a fast advancing field
}

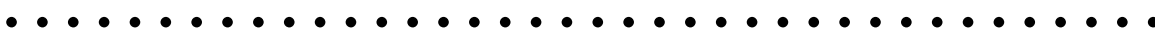

Human molecular genetics 3

Tom Strachan, Andrew P Read. Garland Science, London, New York, 2004

..........

Carla Jodice

European Journal of Human Genetics (2005) 13, 990-991.

doi:10.1038/sj.ejhg.5201432

$\mathrm{T}$ he third edition of Human Molecular Genetics by Strachan and Read provides a most welcome update of an already well established and greatly appreciated Human Genetics text. Such warm reception is due to the fact that Human Molecular Genetics has become an amazingly extraordinary field of study ever since Botstein presented the possibility of developing the first DNA based human gene map in 1980.

Furthermore, over the last 5 years, gathering information on the human genome has become increasingly faster, 
thanks to the availability of new technology and, therefore, new techniques. As a result, our knowledge of the human genome has radically improved. Let us consider, for example, the total number of human genes. Based on a functional evaluation, it was a well established fact shared by the scientific community that the genes contained in the human genome were 80000-100000; the complete sequence demonstrated, instead, that the number of genes is much lower (30000$35000)$ but, surprisingly, code for more than 200000 different transcripts.

Given the most recent discoveries, the present edition has been thoroughly revised and reorganised in four parts.

Part one: The basics. Here the authors describe the fundamentals of Genetics and Molecular and Cellular Biology.

Besides the chapters explaining DNA structure, gene expression, chromosome structure and function, pedigree analysis and those describing the basic, yet latest, laboratory techniques, this third edition has been integrated by a new chapter on cell and development.

This chapter starts with an overview of cell structure and cell diversity and their interactions; it then continues by describing development and morphogenesis. Well-integrated and clear flow diagrams, figures and boxes help the reader to understand the information being presented.

Part two: The human genome and its relationship to other genomes. According to the authors, the second part of this book takes us into the world of genomics or, rather, the foundation of a serious and systematic exploration of the Universe within us. The chapters herein have been rewritten and reorganised, the reason being that in 2003 the sequencing of the human genome and those of five initial model organisms (the bacterium Escherichia coli, the yeast Saccharomices cerevisiae, the roundworm Caenorhabditis elegants, the fruit fly Drosophila melanogaster and the mouse Mus musculus) were completed and are now available on the internet. Furthermore, during the Human Genome Project, other genome projects were undertaken for a wide variety of other model organisms; these studies brought a huge amount of information and thus a new overview needed to be presented.

Part two ends with a new section, which focuses on how comparative analysis of present day genomes have shed light on the evolutionary origin of human DNA and human genes.

Part three: Mapping and identifying disease genes. Here we find a set of chapters describing the various methods of genetic mapping of Mendelian characters, with their advantages and limits, principles and strategies in the identification of disease genes, molecular pathology, cancer genetics and genetic testing in human populations. A chapter is also dedicated to the mapping and identification of genes conferring susceptibility to complex diseases. A considerable number of common diseases, such as diabetes, cancer, Alzheimer's disease, schizophrenia and obesity have been ascertained to be also determined by genetic factors or, at least, that the presence of specific genetic background information confers a higher (or lower) risk to develop the disease.

Being the identification of common diseases susceptibility alleles, the great challenge of the 21st century, the authors well describe the advantages in carrying out research in this field since it could ultimately lead to disease prevention or more rational and targeted approaches to treatment. The authors also discuss the problems involved in such research, such as bias of ascertainment and whether the effort made towards prevention is worthwhile in terms of changes in the lifestyle of the patients.

Part four: New horizons: the 21st century. Here the authors look ahead at the new horizons of functional genomics, transcriptomics and proteomics. A description of Bioinformatics, of the techniques in genetic manipulation of cells and animals and, last but not least, gene therapy, have been obviously thoroughly updated.

In summary, this third edition of Human Molecular Genetics is clear, exhaustive, accurate and up to date in terms of its contents; it has been complemented by a very effective use of colour figures, boxes and flow-charts to help the reader. Each chapter has been completely reorganised so as to present a wealth of information simply and rationally. The use of the internet is also suggested by the authors who, nevertheless, recommend an intelligent and discriminating use of it by supplying reliable core sites as starting points. Further readings and references have been wisely chosen; they include books that the readers will already have and also point to very recent reviews published in easily accessible journals. At the end of the text, a comprehensive, clear, general glossary is provided, including notes referring to other four specialised glossaries. The ideal readers of this book are laboratory scientists, professionals in the medical field and human genetics students who wish to acquire a background knowledge of this fast-advancing field

Carla Jodice is at the Department of Biology, University of Rome 'Tor Vergata', Via della Ricerca Scientifica, 00133 Rome, Italy. E-mail: jodice@uniroma.it 